\author{
Ó. MARTíNEZ-MATOS ${ }^{1, \bowtie}$ \\ G.A. TORCHIA ${ }^{2}$ \\ A. LENCINA ${ }^{2}$ \\ J.O. TOCHO ${ }^{2}$
}

\section{Amplitude gratings generation by selective defect bleaching in $\mathrm{KCl}: \mathrm{CO}_{3} \mathrm{~K}_{2}$ crystals}

\author{
${ }^{1}$ Departamento de Óptica, Facultad de Ciencias Físicas, Universidad Complutense de Madrid, \\ 28040 Madrid, Spain \\ 2 Centro de Investigaciones Ópticas-CIOp (CONICET-CIC) and Universidad Nacional de La Plata, \\ CC 124 La Plata (1900), Argentina
}

\begin{abstract}
Received: 8 October 2007/Revised version: 25 February 2008 Published online: 16 April 2008 • (C) Springer-Verlag 2008
\end{abstract}

ABSTRACT Volume grating formation by spatially-selective defect bleaching is demonstrated in coloured $\mathrm{KCl}: \mathrm{CO}_{3} \mathrm{~K}_{2}$ crystals. The sample is coloured by a train of pulses with a wavelength of $\lambda=266 \mathrm{~nm}$ and bleached with a cw Ar-laser with a wavelength of $\lambda=514.5 \mathrm{~nm}$. Diffraction efficiency for a $1.6 \mu \mathrm{m}$ grating period is studied both experimentally and theoretically, using the evolution of colour centre concentration. Hologram recording parameters are obtained and the evolution of the spatial grating profile is discussed. Diffraction efficiencies three times higher than previously published efficiencies are obtained.

PACS 42.40.Ht; 42.40.Lx; 42.70.Ln

\section{Introduction}

The use of bulk photosensitive materials for storage of massive amounts of information by holographic techniques was first proposed for volume holographic applications over 40 years ago [1]. In the following years the holographic industry developed spectacularly and its application to technologies such as biomedicine [2,3], optical security [4-6], holographic memories [7], optical computing [6,8], optical filters and so on, has been demonstrated successfully. Many studies have been carried out to search for ideal material [6,9-11], for each technological application. In particular, alkaline halides are interesting candidates for holographic applications and they have been studied for the last four decades.

Nominal alkaline halides crystals are insensitive to visible light. Several methods have been proposed to generate photosensitive species in the bulk of the crystals, for example: additive colouring, doping with different cations, electron irradiation, multiphoton absorption and high energy photon absorption. These methods generate colour centers sensitive to visible light. The interaction of these species with light can induce photothermal transformations, molecular reorientations, transformations into higher aggregates or destruction of the centers. Any of these phenomena can be used to record holograms. In this work we use the absorption of high energy photons to generate the colour centers and electron-hole recombination to bleach them.

Fax: +34-91-3944683, E-mail: ommatos@fis.ucm.es
Kogelnik [12] demonstrated in 1969 that phase volume gratings, amplitude volume gratings or both can be used as diffractive elements. These types of holograms have been recorded in alkaline halides crystals. Phase gratings with high efficiency were demonstrated in additively coloured $\mathrm{KCl}$ crystals using the F-X photothermal transformation of colour's centers [13], and phase grating generated by multiphoton absorption has been recorded in LiF by the interference of femtoseconds laser pulses [14]. On the other hand $\mathrm{KCl}, \mathrm{KBr}$ and $\mathrm{NaCl}$ crystals have been used to record amplitude gratings by direct writing of the interference profile of two plane waves. Maximum efficiencies of $0.12 \%$ for $\mathrm{KCl}$ [15], $0.375 \%$ for $\mathrm{KBr}$ [16] and $0.4 \%$ for $\mathrm{NaCl}$ [17] crystals were obtained. These values are far from the theoretical $3.7 \%$ maximum efficiency for this type of grating [12]. Not limited to alkaline halides, amplitude grating generated by the interference of femtosecond laser pulses has been implemented recently in band gap materials [18]. Both amplitude and phase gratings have been recorded simultaneously in $\mathrm{KCl}: \mathrm{Li}$ or $\mathrm{KCl}: \mathrm{Na}$ coloured crystals by the excitation of $\mathrm{F}_{\mathrm{A}}$ centers $[19,20]$.

No experiments have been carried out to record amplitude gratings by spatially-selective bleaching of colour centers in $\mathrm{KCl}: \mathrm{CO}_{3} \mathrm{~K}_{2}$ crystals. In this paper the sample was coloured by a train of pulses with a wavelength of $\lambda=266 \mathrm{~nm}$ and the amplitude gratings have been recorded by two beam interference at an appropriate wavelength. We also propose a model where a defect bleaching mechanism induced by photon absorption is used to study the evolution of a defect density profile during grating recording. Fourier expansion of the spatial defect profile is employed to interpret the efficiency of the first diffraction order.

\section{$2 \quad$ Model concepts}

UV-light irradiation of $\mathrm{KCl}: \mathrm{CO}_{3} \mathrm{~K}_{2}$ crystals induces a cation photoionization and it generates free electrons in the conduction band and holes in the valence band. Free electrons are trapped at anion vacancies to produce F-centers that colour the irradiated crystal. Note that, there is no experimental evidence to relate the F-centre formation with doping concentration in these kind of crystals. Optical bleaching of the defects occurs when the sample is illuminated with light of a wavelength inside the spectral range of the optical absorption of the defects. The trapped electrons are reinduced into the conduction band and electron-hole recombination takes place with efficiency $\gamma$. Then, the F-centers 

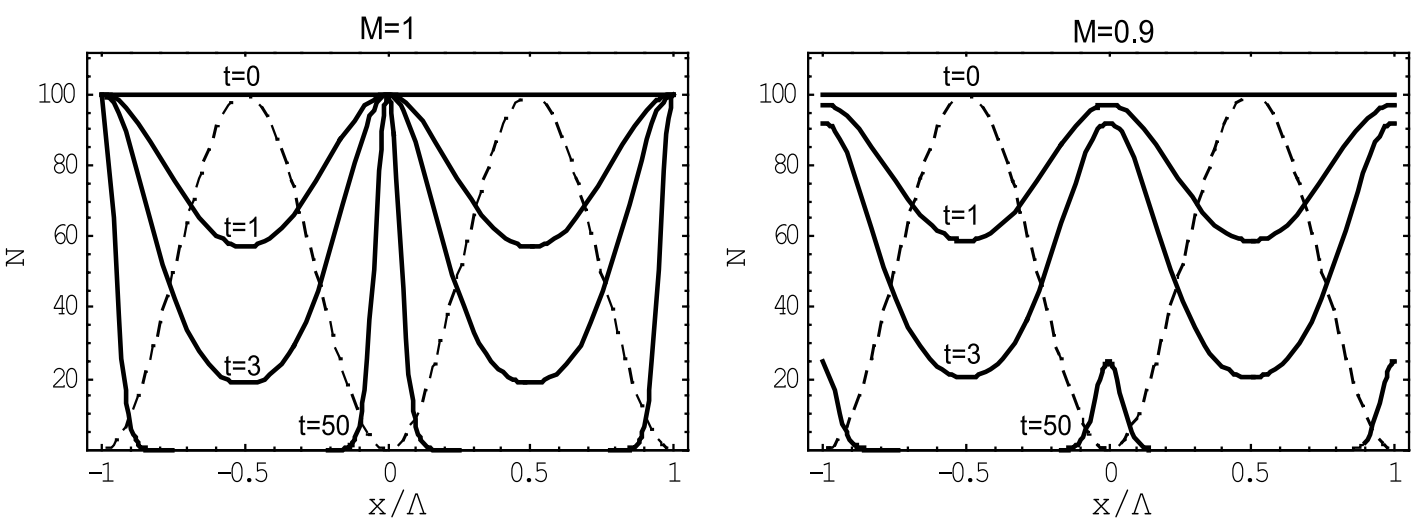

FIGURE 1 Solid lines represent the spatial distribution of defect density at different recording times (in s) for $M=1$ and $M=0.9$. Both figures with $N_{0}=$ 100 and $b=0.28 \mathrm{~s}^{-1}$. The Dashed line represents the normalized intensity pattern

are destroyed and the crystal is bleached. Spatially-selective defect bleaching can be used to record amplitude gratings in a previously irradiated sample. Therefore, the irradiated sample, full of defects, is the starting point for all the experiments we demonstrate here. We assume that colour centers density generated by UV-irradiation is constant for all experiments.

Amplitude gratings were recorded by the interference of two plane waves which generate a periodic intensity pattern:

$I(x)=I_{0}\left(1-M \cos \left(\frac{2 \pi}{\Lambda} x\right)\right)$,

where $I_{0}$ is the sum of the interfering beams intensities; $M$ is the visibility of the interference pattern and $\Lambda$ is the period of the grating. Evolution of defect concentration was studied assuming that the defect bleaching rate is proportional to the light intensity. This assumption is based on the fact that one photon is absorbed by one defect, this induces an electron-hole annihilation and bleaches the F-centre with an efficiency $\gamma$. This optical-bleaching mechanism can be demonstrated in several crystals such as $\mathrm{KH}_{2} \mathrm{PO}_{4}$ (potassium dihydrophosphate or $\mathrm{KDP}$ ), $\mathrm{KD}_{2} \mathrm{PO}_{4}$ (potassium dideuterium phosphate or DKDP) and $\mathrm{NH}_{4} \mathrm{H}_{2} \mathrm{PO}_{4}$ (ammonium dihydrogen phosphate or ADP) in which colour centers are generated by UV irradiation $[21,22]$. The expression that governs the evolution of defects is:

$\frac{\partial N(x, t)}{\partial t}=-\gamma \sigma^{\prime} I(x) N(x, t)$,

where $N(x, t)$ is the defect density and $\sigma^{\prime}=\sigma / \hbar \omega$ the normalized defect absorption cross section. Combining (1) and (2) and solving the differential equation we obtain the evolution of defect density:

$N(x, t)=N_{0} \exp \left(-b\left(1-M \cos \left(\frac{2 \pi}{\Lambda} x\right) t\right)\right)$,

where $b=\gamma \sigma^{\prime} I_{0}$ and $N_{0}$ is the initial defect density of the irradiated $\mathrm{KCl}: \mathrm{CO}_{3} \mathrm{~K}_{2}$ crystal. Note that the parameter $b$ has units of $\mathrm{s}^{-1}$, and then it represents the lifetime of defects for a given illumination. Figure 1 shows the normalized intensity pattern (dashed line) and the spatial distribution of defects (solid lines) for the case of $M=1$ (maxima visibility) and $M=0.9$ for different times with $N_{0}=100$ and $b=0.28 \mathrm{~s}^{-1}$. The defect concentration evolves from a constant value $(t=0 \mathrm{~s})$ to a periodic function dephased by $\pi$ radians regarding the optical intensity distribution. From (2) we can see that the bleaching rate is proportional to $I(x)$, thus low intensity values bleach fewer defects. In the case of $M=1$ defect distribution becomes a set of periodic delta functions when $t \rightarrow \infty$, whereas in the case of $M \neq 1$ defect bleaching located at minimum intensity points (different from zero) takes place and grating contrast will decrease until the hologram is erased completely. As we can see, $M$ is a critical parameter that defines the evolution of the grating contrast along the recording process and determines the quality of the recorded grating.

We can define the absorption coefficient $\alpha(x, t)$ of the irradiated sample to be proportional to the defect concentration $N(x, t)$

$\alpha(x, t)=c N(x, t)$,

where $c$ is a constant with the unit of a cross section. Fourier expansion of the spatial defect concentration (3) is:

$$
\begin{aligned}
N(x, t)= & N_{0} \exp (-b t) \\
& \times\left[J_{0}(\mathrm{i} b M t)+\sum_{n=1}^{\infty}(-\mathrm{i})^{n} 2 J_{n}(\mathrm{i} b M t) \cos \left(\frac{2 \pi n x}{\Lambda}\right)\right] .
\end{aligned}
$$

The spatial defect profile (5) can be interpreted as a superposition of an infinite set of sinusoidal gratings with periods $\Lambda_{n}=\Lambda / n$ ( $n$ is a positive integer), where $J_{n}$ represents the $n$ th-Bessel function of the first kind. The Bragg condition for the $n$-th grating is matched under the incidence angle $\theta_{n}$ of the reading beam. Diffraction efficiencies for volume gratings are very sensitive to $\theta_{n}$ detuning and only the $n$-th grating will contribute to the total diffraction efficiency. The first order diffraction efficiency of an amplitude grating [12] is defined by a mean absorption coefficient $\alpha_{0}(t)$ and a grating amplitude $\alpha_{1}(t)$ associated to the first harmonic order of $\alpha(x, t)$. Experimental conditions were prepared to achieve first order diffraction thus only $\alpha_{0}(t)$ and $\alpha_{1}(t)$ are required.

The combination of (4) and (5) enable us to find the required expressions for $\alpha_{0}(t)$ and $\alpha_{1}(t)$ : 
$\alpha_{0}(t)=a \exp (-b t) J_{0}(\mathrm{i} b M t)$

$\alpha_{1}(t)=-2 \mathrm{i} a \exp (-b t) J_{1}(\mathrm{i} b M t)$,

where $a=c N_{0}$ and represents an absorption coefficient proportional to the number of initial defects $N_{0}$.

Figure 2 shows the results of the model for the zero and first order absorption coefficients for a bleaching absorption grating recorded under the same conditions as shown in Fig. 1. Parameter $a$ is set to $0.79 \mathrm{~cm}^{-1}$. Note that first order diffraction efficiency is composed of two symmetrical diffraction directions ( +1 and -1 orders), thus $\alpha_{1}(t) / 2$ is the weight of the total absorption coefficient to each diffraction direction. The same conclusion is obtained expressing (4) in complex notation and interpreting each complex exponential as the contribution to +1 or -1 directions of the diffracted beam. It can be observed from Fig. 2 that $\alpha_{0}(t)$ decreases with recording time as defects are bleached. This result indicates that $\alpha_{0}(t)$ is proportional to the total number of defects, as we proposed in (4). However, $\alpha_{1}(t) / 2$ increases reaching its maximum value (grating formation) and behaves as $\alpha_{0}(t)$ for long recording times. Simulation for $M=1$ and $M=0.9$ (Fig. 2) shows slight differences for the behaviour of $\alpha_{0}(t)$ and $\alpha_{1}(t) / 2$; however these differences cause substantial changes in diffraction efficiency as will be discussed below. Gratings recorded with maximum visibility $(M=1)$ survive for a longer time as the defect distribution becomes a set of periodic delta functions when $t \rightarrow \infty$.

The diffraction of an amplitude grating was studied by Kogelnik [12]. He found that the first order diffraction efficiency $\eta$ of an isolated sinusoidal amplitude grating for the on-Bragg condition is:

$\eta(d, t)=\exp \left(-\frac{2 \alpha_{0}(t) d}{\cos (\theta)}\right) \sinh ^{2}\left(\frac{\alpha_{1}(t) d}{2 \cos (\theta)}\right)$,

where $d$ represents the depth of the grating and $\theta$ is the semiangle between the recording beams inside the sample. Expression (8) predicts a maximum diffraction efficiency of $3.7 \%$ when conditions $\alpha_{0} / \alpha_{1}=1$ and $\alpha_{1} d / \cos (\theta) \approx 1.1$ are satisfied simultaneously. This expression together with (6) and (7) will be used to fit experimental results. Note that the grating depth $d$ must be corrected to take into account the finite size of the writing beams, which is usual when the superposed beams give rise to a grating whose depth is smaller than the crystal

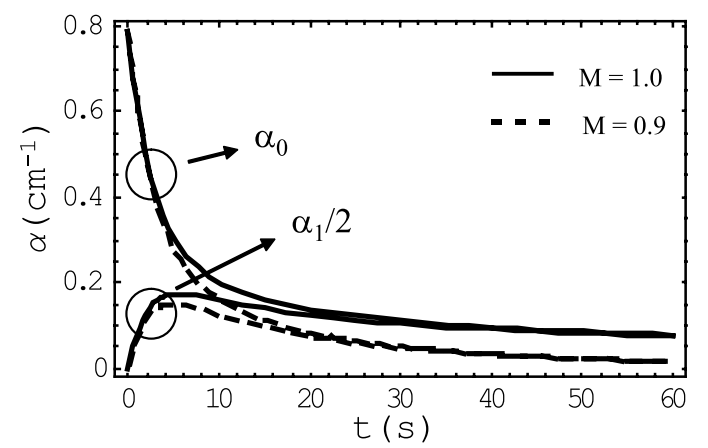

FIGURE 2 Zero $\alpha_{0}$ and first $\alpha_{1} / 2$ order Fourier expansion of the absorption coefficient $\alpha$ for $M=1.0$ and $M=0.9$. All curves with $N_{0}=100$ $a=0.79 \mathrm{~cm}^{-1}$ and $b=0.28 \mathrm{~s}^{-1}$ depth [23]. However, the transmittance of one of the writings beams during recording is, as usual:

$T(t)=\exp \left(-2 \alpha_{0}(t) d / \cos (\theta)\right)$

However, to be accurate, the intensity of the readout beam must be corrected considering losses before it reaches the grating.

\section{Experimental results and discussion}

Experimental work has been done using a $\mathrm{KCl}$ crystal of size $10 \times 10 \times 6.4 \mathrm{~mm}^{3}$, doped with $\mathrm{CO}_{3} \mathrm{~K}_{2}$ at $0.01 \%$ concentration. No colour centers were observed in the sample before UV-irradiation. The crystal became coloured when the sample was irradiated with a train of $\lambda_{\mathrm{C}}=266 \mathrm{~nm}$ pulses coming from the fourth harmonic of a Nd-YAG pulsed laser with $10 \mathrm{~ns}$ pulse width. UV-irradiation was maintained on until the transmitted intensity became stable, so obtaining a uniform coloured sample. This condition was the starting point for all experiments. Absorption spectrum of the sample after irradiation is shown in Fig. 3. We observe a wide absorption band, from $\lambda=500 \mathrm{~nm}$ to $\lambda=700 \mathrm{~nm}$ that is associated with the presence of F-centers.

We have recorded transmission volume holographic gratings by the interference of two mutually coherent $s$-polarized collimated writing beams from an Ar-laser with a wavelength of $514.5 \mathrm{~nm}$, a mean intensity $I_{0}=60 \mu \mathrm{W}$ and a crossing angle of $2 \theta=12.5^{\circ}$ inside the medium. The resulting grating spacing was thus $1.6 \mu \mathrm{m}$. Defect bleaching was easily achieved since the writing beams wavelength fall in the tail of the F-centre absorption spectrum. We also use the $s$-polarized collimated Ar-laser beam to read the hologram by blocking one of the two recording beams. Thus the reading beam was under the Bragg condition and first-order diffraction intensity comes from the first-order amplitude absorbing coefficient $\alpha_{1}$. Illuminating the sample with just one beam erases the grating, therefore, this medium is reversible and different holograms were recorded on the same sample. Thermal bleaching of the centers during optical exposure was negligible and the holograms mean life is approximately 40 days.

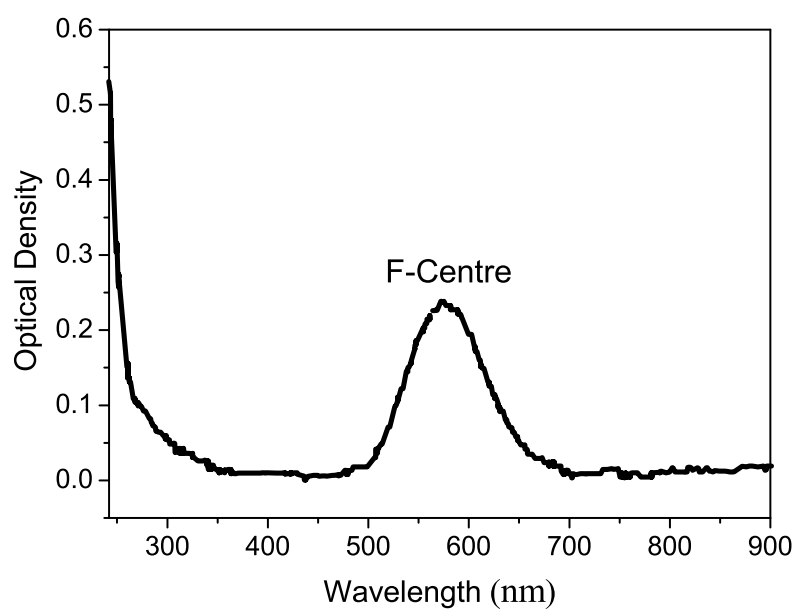

FIGURE $3 \mathrm{KCl}: \mathrm{CO}_{3} \mathrm{~K}_{2}$ absorption spectrum after UV-irradiation. An absorption band due to F-centers can be observed 


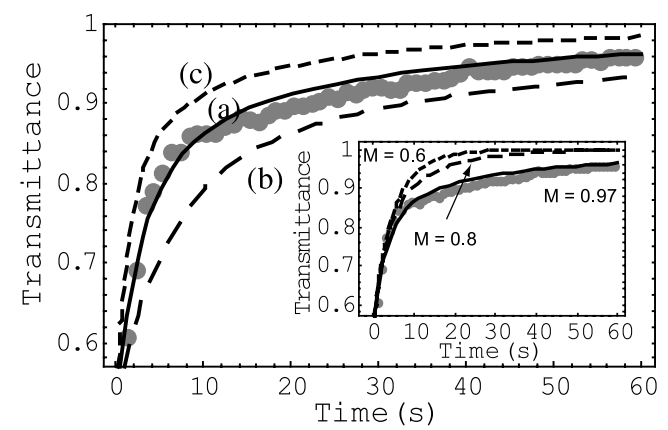

FIGURE 4 Transmittance of one of the writing beams during grating recording. Full circles correspond to experimental results. Continuous line (a) best fit using (6) and (9), which gives $a=0.79 \mathrm{~cm}^{-1}, b=0.28 \mathrm{~s}^{-1}$ and $M=0.97$; dashed lines: model results for non-optimized $b$ values (b) $b=0.14 \mathrm{~s}^{-1}$ (c) $b=0.56 \mathrm{~s}^{-1}$. Inset: Transmittance versus time for $a=$ $0.79 \mathrm{~cm}^{-1}, b=0.28 \mathrm{~s}^{-1}$ and $M=0.97$ (solid line), $M=0.8$ and $M=0.6$ (dashed lines)

Two experiments were carried out to characterize the evolution of the grating and to validate the proposed model. First, we studied the transmittance of the grating for one of the writing beams during recording with the aim of finding $a, b$ and $M$ by means of (9). These parameters were used to characterize $\alpha_{0}(t)$ and $\alpha_{1}(t)$ using (6) and (7). Second, by measuring the diffraction efficiency for different recording times and by using the expressions $\alpha_{0}(t)$ and $\alpha_{1}(t)$ found previously and (8), the validity of the model was checked.

Transmittance for one of the writing beams during recording is shown in Fig. 4. Full circles are the experimental results and the solid line (a) is the best fit using (9) and (6) which gives $a=0.79 \mathrm{~cm}^{-1}, b=0.28 \mathrm{~s}^{-1}$ and $M=0.97$. Good fitting with less than $10 \%$ error is obtained for $a=(0.75 \pm 0.10) \mathrm{cm}^{-1}$, $b=(0.25 \pm 0.03) \mathrm{s}^{-1}$ and $M=(0.97 \pm 0.03)$. Dashed lines display the model results for non-optimized $b$ values $(b=$ $0.14 \mathrm{~s}^{-1}$ and $b=0.56 \mathrm{~s}^{-1}$ for curves (b) and (c), respectively). Note that, more than $35 \%$ of the incident intensity was absorbed at the initial stage $(t=0 \mathrm{~s})$. Transmittance growths quickly and it asymptotically reaches unity. For long times the sample was completely bleached and there were no remnant defects. From the results of the model we observe that small changes in $b$ give rise to intolerable fittings indicating the sensibility of the model to one of the following parameters: defect absorption cross section, bleaching coefficient or beam intensity. In the inset of Fig. 4 we also demonstrate the sensibility of the model to small changes in $M(M=0.8, M=0.6)$. Maximum transmittance (unity) is reached at short times when visibility is low, indicating a fast defect bleaching. This simple experiment enables us to characterize parameter values $a$, $b$ and $M$ and the coefficients $\alpha_{0}(t)$ and $\alpha_{1}(t)$ that define grating formation.

The validity of the proposed model was checked by analyzing the diffraction efficiency of the recorded gratings. In Fig. 5 the first order diffraction efficiency of the amplitude gratings for different recording times is shown. Each experiment is represented by a full circle and it was obtained as follows: an amplitude grating was recorded in the crystal during the time indicated in the abscises coordinate; then, one of the recording beams was blocked and the diffraction efficiency was measured; after that, the amplitude grating was erased completely, the sample was UV-re-irradiated and a new holo-

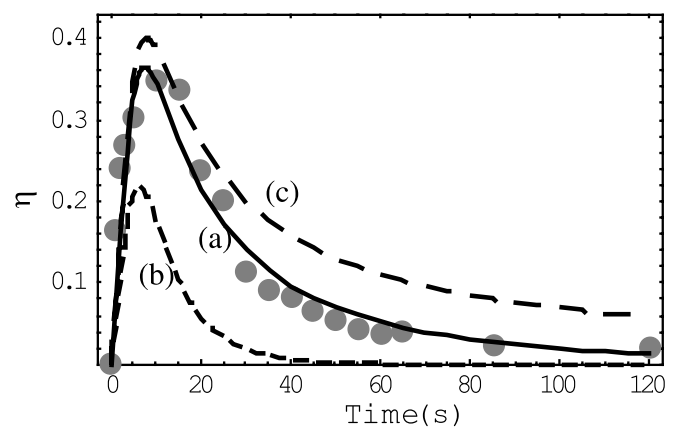

FIGURE 5 First order diffraction efficiency of an amplitude grating for different recording times. Full circles correspond to experimental data obtained from each grating as explained in the text; continuous lines correspond to: (a) best fit with (6)-(8) which gives $a=0.82 \mathrm{~cm}^{-1}, b=0.22 \mathrm{~s}^{-1}$ and $M=0.97$; dashed lines represent the model results for non-optimized $M$ values (b) $M=0.8$ and (c) $M=1.0$

gram was registered with a different recording time, and so on. Thus, each point represents the result obtained for an individual grating. The measured efficiency increases quickly with the recording time, reaching a maximum value of $\eta=0.35 \%$ and then decrease relatively slowly to zero when $t \rightarrow \infty$. The maximum efficiency obtained is far from the theoretical maximum which is $3.7 \%$, but matches very well with the predictions of the proposed model. This low efficiency can be explained noticing that the conditions for maximum efficiency, $\alpha_{0} / \alpha_{1}=1$ and $\alpha_{1} d / \cos (\theta) \approx 1.1$, are not satisfied simultaneously. The best fit give rise to $\alpha_{1} d / \cos (\theta)<1$.1. For a fixed experiment in which $d$ and $\theta$ are constants, $\eta$ will be improved only when defect concentration at the initial stage $\left(N_{0}\right)$ is high enough as to increase the value $\alpha_{1}$, see (7). This condition can be achieved irradiating the sample with X-ray or using high energy UV-lasers. Nevertheless, $\eta=0.35 \%$ is three times better than any other value obtained for amplitude gratings in $\mathrm{KCl}$ crystals. The improvement of the diffraction efficiency comes from the recording method employed. The $\mathrm{cw}$-laser used to bleach the defects can be collimated in a large region and the resulting interferometric pattern has very high quality. Note that other authors use the interference of UVpulses to record the amplitude gratings by a direct writing method. The interferometric pattern obtained by this method has lower quality due to two main reasons: the experimental difficulties in obtaining collimated UV beams and also the wider spectra range of pulsed lasers. Those setbacks induce lower visibility $M$ on the interferometric pattern and the diffraction efficiency decreases, as it is shown in Fig. 5. The solid line in Fig. 5 is the best fit to experimental results by using (6)-(8). The dashed lines $(b, c)$ were calculated with the help of the model for non-optimized $M$ values. In all cases, as shown in the figure $a=0.82 \mathrm{~cm}^{-1}$ and $b=0.22 \mathrm{~s}^{-1}$. Visibility values $M=0.97, M=0.8$ and $M=1$ correspond to curves (a), (b) and (c), respectively. As can be seen in the figure, efficiency is very sensitive to small changes in grating visibility $M$. High quality gratings are obtained for $M=0.97$ and a recording time of about $18 \mathrm{~s}$. Longer exposure times bleach the grating at a rate that depends on the visibility value. Model results show that gratings with $M=1$ survive longer exposure times since there are defects placed at zero intensity values that can not be bleached. Note that best fit values 
$a=0.82 \mathrm{~cm}^{-1}, b=0.22 \mathrm{~s}^{-1}$ and $M=0.97$ are within the reliable interval found in Fig. 4. This fact confirms the validity of the proposed model and diffraction efficiency for these kind of amplitude gratings can be interpreted.

\section{Conclusions}

In this paper we have recorded amplitude gratings in $\mathrm{KCl}: \mathrm{CO}_{3} \mathrm{~K}_{2}$ crystals which were coloured by a train of pulses with a wavelength of $\lambda=266 \mathrm{~nm}$ by F-centre bleaching through a two beam interference pattern at $\lambda=514.5 \mathrm{~nm}$. Volume grating formation of a $1.6 \mu \mathrm{m}$ spatial period has been demonstrated at room temperature and holograms mean life is approximately 40 days. To our knowledge that is the first time that an amplitude grating was recorded by spatially-selective bleaching of colour centers in $\mathrm{KCl}$ crystals. Moreover, we proposed a model to find the mean absorption coefficient $\alpha_{0}(t)$ and the amplitude absorption coefficient $\alpha_{1}(t)$ associated with the first diffraction order of the amplitude grating. To do this, we considered the absorption coefficient to be proportional to the defect concentration $N(x, t)$ and the bleaching defect rate to be proportional to the intensity of the recording light. Then, hologram recording parameters were obtained and the evolution of the spatial grating profile could be interpreted. Experimental and theoretical investigation of amplitude gratings in $\mathrm{KCl}$ crystals open new possibilities for controlling amplitude grating or mixing amplitude-phase gratings in any kind of holographic material in which colour centers are involved.

ACKNOWLEDGEMENTS This work was partially supported by the Spanish Ministry of Education and Science under project TEC2005-
02180, by ANPCyT (Argentina) under project PICT-15210 and by CONICET (Argentina) under project PIP 5997.

\section{REFERENCES}

1 P.J. Van Herden, Appl. Opt. 2, 393 (1963)

2 O. Coquoz, R. Conde, F. Taleblou, C. Depeursinge, Appl. Opt. 34, 7186 (1995)

3 F. Fercher, W. Drexler, C.K. Hitzenberger, T. Lasser, Rep. Prog. Phys. 66, 239 (2003)

4 J.L. Horner, B. Javidi, Opt. Eng. 35, 2451 (1996)

5 P. Refregier, B. Javidi, Opt. Lett. 20, 767 (1995)

6 M.L. Calvo (Coord.), Optica Avanzada (Ariel, serie Ciencia, Barcelona, 2002)

7 H.J. Coufal, D. Psaltis, G.T. Sincerbox, Holographic Data Storage (Springes Series in Optica Sciences 76, 2000)

8 H.J. Coufal, Proc. SPIE 5005, 439 (2003)

9 W.S. Colburn, J. Imaging Sci. Technol. 41, 443 (1997)

10 L. Solymar, D.J. Cooke, Volume Holography and Volume Gratings (Academic, London, 1981)

11 F.T. O'Neill, J.R. Lawrence, J.T. Sheridan, Appl. Opt. 41, 845 (2002)

12 H. Kogelnik, Bell. Syst. Tech. J. 48, 2909 (1969)

13 D.A. Vladimirov, V.E. Mandel, A.Yu. Popov, A.V. Tyurin, Opt. Spectrosc. 99, 137 (2005)

14 K.-I. Kawamura, D. Takamizu, T. Kurobori, T. Kamiya, M. Hirano, H. Hosono, Nucl. Instrum. Methods Phys. Res. B 218, 332 (2004)

15 B. Stadnik, Z. Tronner, Nouv. Rev. Opt. Appl. 3, 347 (1972)

16 A.A. Friesem, J.L. Walker, Appl. Opt. 9, 201 (1970)

17 O. Salminen, R. Hamalainen, P. Ketolainen, T. Keinonen, Opt. Acta 25, $285(1978)$

18 S.M. Avanesyan, S. Orlando, S.C. Langford, J.T. Dickinson, Proc. SPIE 5352, 169 (2004)

19 M. May, J.P. Hong, S. Debrus, J. Opt. Soc. Am. B 5, 2333 (1988)

20 D. Chen, Proc. IEEE 63, 1207 (1975)

21 C.D. Marshall S.A. Payne, M.A. Henesian, J.A. Speath H.T. Powell, J. Opt. Soc. Am. B 11, 774 (1994)

22 O. Martínez-Matos, G.A. Torchia, G.M. Bilmes, J.O. Tocho, H.F. RaneaSandoval, Appl. Phys. B 80, 535 (2005)

23 M. Tebaldi, A. Lencina, N. Bolognini, Opt. Commun. 220, 257 (2002) 